\title{
31. Posttraumatic stress in Covid 19 patients after extubation in an intensive care unit (ICU)
}

Vasileios Flampouraris $^{1, *}$, Maria Kokolaki ${ }^{1}$, Stella Kandreviotou ${ }^{1}$, Anastasia Niaka ${ }^{1}$, Andromachi Papadopoulou ${ }^{1}$

1"Sismanoglio- Amalia Fleming" General Hospital, Department of Anaesthesiology and Pain Medicine, Athens, Greece.*vasflamp@gmail.com

If you're patient in an ICU, this fact is in itself a traumatic unpleasant event.

Covid 19 patients who admitted to the intensive care unit urgently, are provided with a wide range of machines for safe monitoring.

Objective-purpose: The purpose is to record the physical and emotional unpleasant experience during their hospitalisation in ICU.

Material-methods: We conducted a study of 18 patients, (44 to 82 years old), ASA II-III, 12 men and 6 women. We filled out a questionnaire.

1. What was the difficulty in communication?

2. What was scary in the environment (ICU)?

3. Do you suffer from insomnia? (noises from ventilators or monitors).

4. Do you feel pain ?(VAS scale)

5. Do you feel muscle weakness?

6. Do you know where are you ?(disorientation)

7. Do you want to talk to your family?

8. Emotional changes (anger, aggression, arousal.)

9. Can you swallow?

Results: (8) Covid 19 patients difficulty in communication. (14) Covid 19 patients were scary. (12) Covid 19 patients suffered from insomnia. (16) Covid 19 patients felt pain VAS $>8$. (18) Covid 19 patients felt weakness. (11) Covid 19 patients with disorientation. (6) Covid 19 patients with emotional changes (anger, aggression, arousal). (12) Covid 19 patients: difficulty swallowing. Symptoms such as muscle weakness or insomnia persist 6 months after discharge from the hospital.

Conclusions: Memories of posttraumatic stress in Covid 19 patients in an ICU appear to be different, unprecedented and particularly stressful. These patients need a long term psychological support ie desensitisation. A plan is required : I start from the beginning to live. 